\section{Post-traumatic stress symptoms in pediatric heart transplant recipients}

\author{
Elana E. Evan, Payal A. Patel, \\ Alison Amegatcher, Nancy Halnon \\ Department of Pediatric Cardiology, \\ University of California, Los Angeles, CA, \\ USA
}

\section{Abstract}

Traumatic experiences are not unusual in pediatric heart transplant (HT) recipients before and after transplantation. Post-traumatic stress symptoms (PTSS) present at the time of transplant evaluation and developing afterward occur with an unknown frequency. We sought to determine the burden of these symptoms in heart transplant patients. We reviewed 51 consecutive HTs between 2003-2007, including 40 primary transplants and 11 retransplants. Symptoms were present in 17 of the 51 patients (34\%) at the time of orthotopic heart transplantation evaluation. None met the criteria for full post traumatic stress disorder. Transplant complications were examined. Nineteen subjects of the total sample had rejection in the first year following transplant. Rejection rates in the first year was $41 \%$ for those with PTSS (7 of 17 patients) and 36\% for those without (12 of 33 patients) ( $\mathrm{P}=\mathrm{n} . \mathrm{s})$. Of those patients presenting for a second heart transplant, 55\% had PTSS at the time of transplant evaluation and/or the peritransplant period; whereas, (28\%) undergoing a primary transplant had PTSS. In addition to symptoms resulting from the disease process leading to HT and other prior experiences, the HT itself seems to present a large psychiatric burden on patients. All patients need to be followed before and after HT for signs and symptoms related to PTSS. Future studies should be undertaken to determine if preventative detection and treatment of patients with these PTSS symptoms early can lead to better outcomes.

\section{Introduction}

With continued improvement in medical outcomes of pediatric heart transplantation (HT), there is increasing interest in understanding the impact of transplantation and post-transplant care on the psychological functioning of these recipients. The challenges of life-long medical visits, adherence to medications, and procedures to monitor rejection after solid organ transplant places undue stress on the patient and their families. ${ }^{1}$

Heart failure results from a variety of congenital and acquired cardiovascular diseases. It is one of the most common chronic conditions affecting adults, especially the elderly. However, many children and adolescents with heart failure, first present for medical care with severe symptoms already at end stage. Many are hemodynamically compromised requiring intensive care or invasive therapies such as placement of ventricular assist devices or extracorporeal support prior to medical stabilization with heart transplantation. Traumatic experiences, then, are not unusual in pediatric heart transplant recipients. Mintzner et al. found that $30 \%$ of adolescents who underwent solid organ transplant displayed symptoms of stress, from posttraumatic stress symptoms (PTSS) to post-traumatic stress disorder (PTSD). ${ }^{2}$ Previous work by investigators in the area of PTSD has examined medicalized children. ${ }^{3,4}$ However, lesser degrees of psychiatric symptoms and disease states still may have a critical effect on the well being of children with chronic disease (such as heart transplant recipients), but have been minimally explored. In this retrospective chart review, we investigated the frequency of PTSS during the transplant evaluation and peritransplant periods in first time and repeat transplant recipients, as well as short and long term outcomes. Additionally, we investigated whether the rates of rejection or death differ in those pediatric heart transplant patients that experience PTSS compared to those without these symptoms. The goal of this study is to establish a framework to explore potential avenues for further investigation into the impact of posttraumatic stress symptoms in pediatric acute and chronic illness and transplantation.

\section{Materials and Methods}

\section{Selection of subjects and clinical characteristics}

This study was conducted with approval of the institutional review board for the University of California, Los Angeles (USA). A retrospective chart review was conducted of hospital records, operative notes, and outpatient notes of all pediatric patients ( 0 to 20 years) who underwent orthotopic heart transplantation at our institution over a three year period from January 2004 through February 2007 and subsequent follow up. We reviewed medical history to ascertain underlying cardiac diagnosis, occurrence of rejection, and posttraumatic stress symptoms reported by patients to practitioners. Clinic visits up to one year post-transplant were included in the chart review and long term outcome including death or graft loss by three to five years post-transplant was recorded. Encounters included those
Correspondence: Elana E. Evan, Mattel Children's Hospital and David Geffen School of Medicine at UCLA, MDCC \#22-464, 10833 LeConte Ave., Los Angeles, CA 90095, USA.

E-mail: eevan@mednet.ucla.edu

Key words: posttraumatic stress symptoms, pediatric heart transplant, rejection.

Contributions: EEE, concept and design of the project, critical revision of the article, drafting of the manuscript and statistics; PAP, data collection and drafting of the article; $\mathrm{AA}$, data collection and critical revision of the article; $\mathrm{NH}$, concept and design of the project, drafting of the manuscript, critical revision of the article, and statistics.

Conflict of interests: the authors declare no potential conflict of interests.

Received for publication: 4 April 2013.

Accepted for publication: 12 April 2013.

This work is licensed under a Creative Commons Attribution NonCommercial 3.0 License (CC BYNC 3.0).

(C) Copyright E.E. Evan et al., 2014

Licensee PAGEPress, Italy

Health Psychology Research 2014; 2:1549

doi:10.4081/hpr.2014.1549

for both the routine pre-transplant psychosocial evaluation and additional consultations requested by patients and or practitioners for the evaluation and treatment of behavioral complaints or concerns for psychiatric involvement. Evaluating practitioners included clinical social workers (who perform routine pretransplant evaluations) and child psychiatrists or psychologists consulted when concerns for psychiatric pathology were present. Charts were reviewed for any post-traumatic stress symptoms listed under the DSM-IV's diagnostic criteria for PTSD including: re-experiencing, avoidance, and hyperarousal symptoms as documented in psychiatric and social work notes prior to transplant. According to the DSM-IV, 5 re-experiencing was defined as repetitive play, anxiety, or nightmares. Hyperarousal symptoms were defined as increased startle, irritability, or insomnia. Social impairment was defined as not playing with others or poor adjustment in school. Avoidance symptoms included detachment or regression in development. For the purpose of this retrospective chart review, patients with at least one symptom in any category at any time (prior to or within one year of transplant) were considered to have PTSS. Patients were considered to have had a rejection episode if a biopsy or autopsy showed histologic evidence of humoral or cellular rejection, treatment was given for clinically suspected rejection with 
subsequent improvement in symptoms, or death within one year of transplant when no autopsy was performed.

\section{Statistical analysis}

Frequency of PTSS was treated as a categorical variable for analysis. Categorical variables were analyzed using the Pearson chi-square test. Continuous variables were analyzed using the t-test. For demographics (gender, age, religion, ethnicity, PTSS, and caregivers), frequency was calculated. We also compared frequency of post-traumatic stress symptoms and rejection (as defined above). All statistical tests were two-sided, and a $\mathrm{P}<0.05$ was considered significant. The analyses were performed using PASW Statistics-Version 18.0.

\section{Results}

\section{Characteristics of the study population}

Between January 2004 and 2007, 59 children and adolescents under 21 years old received heart transplants at our institution. We excluded all patients under the age of 2 years, as these patients are difficult to evaluate for psychiatric illness. After excluding these patients, our cohort had 51 patients, including 40 primary transplants and 11 re-transplants. The mean age for the entire cohort was 12.3 years with mean age at the time of primary transplant 11 years (range 2 to 18 years), and for retransplant mean age was 14.6 years of age (range 9-20 years). Average wait time for an organ for the entire cohort was 49 days, with a wait time of 37.8 days for the primary transplants, and 70.9 days for the retransplants. In both the primary transplant and retransplant groups, males and females were evenly distributed (20 and 20 vs. 5 and 6 respectively). Age, gender and ethnicity of our entire cohort is summarized in Table 1.

\section{Pretransplant incidence of post traumatic stress symptoms}

We examined the incidence of PTS-related symptoms present at the time of heart transplantation, specifically those noted in the peritransplant period. Post traumatic stress symptoms were present in 22 of 51 patients (43\%) at the time of HT evaluation. No patients met the criteria for a clinical diagnosis of PTSD. According to the Diagnostic and Statistical Manual (DSM) of Mental Disorders, Version 4,5 patients with PTSD fall into 3 symptom clusters: re-experiencing, avoidance, and arousal. A clinical diagnosis of PTSD requires that the patient have symptoms for at least a month, and have one re-experiencing symptom, three avoidance symptoms, and two hyperarousal symptoms. Re-experiencing symptoms include recurrent and distressing recollections of the event, repeated distressing dreams of the event, acting flashbacks, intense psychological distress at exposure to reminders, and physiologic reactivity to reminders. Avoidance symptoms consist of efforts to avoid thoughts or places associated with the traumatic event, inability to recall important aspects of the event, diminished interest in activities, feelings of detachment from others, restricted range of affect, and a sense of foreshortened future. Increased arousal is characterized by difficulty falling or staying asleep, irritability, difficulty concentrating, hypervigilance, and exaggerated startle response. No patients in our chart review met diagnostic criteria for PTSD; however, any patient that was documented as having at least one symptom from any diagnostic category was considered to have PTSS. In our sample 17 patients (34\%), 1 or more post-traumatic stress symptom. Some patients had more than one of each type of symptom. For instance, a patient could have nightmares and anxiety (both symptoms of reexperiencing) as well as irritability, which is part of increased arousal. Interestingly, we noted the presence of other co-morbid psychiatric symptoms in our sample, and found that depressive symptoms were also relatively high (22\%) in pediatric patients in the pre-evaluation and peritransplant phase of heart transplantation (Table 2).

\section{Posttraumatic stress symptoms in patients undergoing repeat trans- plantation}

We found no significant differences between the frequency of post-traumatic stress symptomsin patients requiring re-transplant compared to those undergoing a primary transplant. However, we noted that out of the 11 subjects with prior transplant, 6 (55\%) had PTSS at the time of transplant evaluation and in the peritransplant period, 5 (45\%) did not. Of the 11 with a prior transplant, only 2 had depression and both of these patients had PTSS as well. The frequency of prior transplant patients with PTSS gives us an opportunity to think about the long term effects of the transplant on the patients' mental well being.

\section{Table 1. Patients characteristics.}

\begin{tabular}{lc} 
Subject age (years \pm SEM) & $12.3 \pm 0.63$ \\
Gender (male : female) & $25: 25$ \\
\hline Average wait time (days \pm SEM) & $49 \pm 6.93$ \\
Medial history (at time of transplant evaluation) & \\
Prior heart transplant & $11(22 \%)$ \\
Congenital heart disease & $12(24 \%)$ \\
Chronic illness affecting other than the heart & $24(48 \%)$ \\
\hline Ethnicity (self-reported) & \\
Caucasian & $17(34 \%)$ \\
Hispanic & $16(32 \%)$ \\
African-American & $8(16 \%)$ \\
Pacific Asian & $5(10 \%)$ \\
Latino & $2(4 \%)$ \\
Armenian & $1(2 \%)$ \\
Missing/not stated & $9(18 \%)$ \\
Religion & \\
Catholic & $13(26 \%)$ \\
Christian & $5(10 \%)$ \\
Baptist & $4(8 \%)$ \\
Episcopal & $2(4 \%)$ \\
Lutheran & $2(4 \%)$ \\
Methodist & $1(2 \%)$ \\
Jewish & $1(2 \%)$ \\
Mormon & $1(2 \%)$ \\
Non-believer & $1(2 \%)$ \\
Missing/not stated & $20(40 \%)$ \\
\hline Primary caretaker for medical needs & \\
Mom & $16(32 \%)$ \\
Dad & $1(2 \%)$ \\
Mom and Dad & $18(36 \%)$ \\
Grandmother & $2(45)$ \\
Grandmother and Mom & $1(2 \%)$ \\
Sister & $2(4 \%)$ \\
Unknown & $10(20 \%)$ \\
Family & \\
Father uninvolved & $9(18 \%)$ \\
\hline & $3(6 \%)$ \\
\hline & \\
\hline & \\
\hline
\end{tabular}




\section{Frequency of rejection and death}

We also sought to determine if the presence of post-traumatic stress symptoms had an impact on transplant complications by examining the frequency of death and rejection in the cohort. Nineteen patients had rejection based on our criteria within the first year. Among 33 patients without PTSS, 12 (36\%) had rejection and $21(64 \%)$ had no rejection. Seven of the 17 patients (41\%) with PTSS had rejection and 10 (59\%) had no rejection $(\mathrm{P}=\mathrm{n} . \mathrm{s})$.

\section{Discussion and Conclusions}

Few studies have examined incidence or effects of PTSS in pediatric heart transplant recipients. The pediatric heart transplant recipients at our institution are often first encountered in the end stages of heart failure, requiring assist devices and other invasive procedures to bridge the patients until transplantation. Undoubtedly, this is very traumatic to the patient and family, and not surprising that over one-third of the patients in our cohort displayed symptoms of PTSS. Mintzner et al. found that $30 \%$ of adolescents who underwent solid organ transplant displayed symptoms of stress, from PTSS to PTSD.2

Avoidant symptoms as part of PTS may contribute to non-adherence. ${ }^{6}$ Because adherence could not be rigorously measured in this retrospective study, we extrapolated for the presence of this behavior using frequency of rejec- tion. Although we found that many of our patients had both posttraumatic stress symptoms and rejection, we did not find an association between the two. This lack of association between symptoms and rejection could have been attributed to a number of factors including a small sample size and lack of a formalized assessment protocol of psychiatric symptomology. Even though our pretransplant evaluation is thorough, involving a multidisciplinary team, psychiatry consults are not done on every patient, particularly younger, preverbal patients. While we did not find an association of PTSS with death or rejection, other variables leading to poor outcomes, such as adherence to treatment, could be affected by psychiatric symptoms. Further studies designed to capture these data would be required.

At the outset, we hypothesized that pretransplant illness may be a major contributor to symptoms. The significant number of patients in this cohort receiving repeat transplants allowed us to examine effects of the transplant process itself on incidence of PTSS. Although we observed that patients undergoing retransplant displayed more post-traumatic stress symptoms prior to the second HT than their counterparts undergoing primary HT, the difference was not statistically significant. Still, the frequency of traumatic stress symptoms observed through this chart review prompts further investigation. Rather than the disease process leading to HT representing the most significant stressor, the HT itself seems to present a large psychiatric burden on

Table 2. Patients history.

\begin{tabular}{lc} 
Symptoms & N. (\%) \\
History of psychiatric symptoms and diagnoses & $11(22)$ \\
Depression & $4(8)$ \\
Psychosis or delerium & $2(4)$ \\
Autism & $1(2)$ \\
Anorexia /cutting & $1(2)$ \\
Acute stress disorder, nos & $5(10)$ \\
Risky behaviors* & $17(34)$ \\
Symptoms associated with posttraumatic stress occurring in the peri-tranplant period & $13(26)$ \\
Patients with 1 or more symptom related to PTS & $2(4)$ \\
\hline Frequency of patients with specific symptoms & $13(26)$ \\
Reexperiencing & $3(6)$ \\
Repetitive play & $11(22)$ \\
Anxiety & $3(6)$ \\
Nightmares & $6(12)$ \\
Hyperarousal & $10(20)$ \\
Startle & $2(4)$ \\
Irritablity & $3(6)$ \\
Insomnia & $11(22)$ \\
Impairment & $1-8$ \\
Avoidance & \\
Patients with $>2$ symptoms & \\
Number of symptoms present in those patients with post traumatic stress-related & \\
symptoms (range) & \\
\hline *Risky behaviors consist of smoking & \\
&
\end{tabular}

patients. Other factors may also contribute. Retransplant patients had longer wait times. These patients tended to be older, in the adolescent age group, and studies have shown that this group of patients are the most nonadherent.2,7,8 Our results lead us to believe that a future larger prospective study aimed at assessment of and evaluating effects of interventions for PTSS both at the time of transplant and at intervals after transplant would be beneficial.

This study is subject to many limitations. We performed a retrospective chart review without the advantages of prospective data collection and a validated instrument such as those utilized for the assessment of PTSD. ${ }^{9}$ Clinical notes cannot provide reliable diagnoses best employed in research studies. It would not be possible to determine causative factors related to posttraumatic stress symptoms, as some symptoms noted in patients in this cohort may be found even in normal children. Through this chart review, we had no method to determine the impact of cognitive impairment known to be present in some children after cardiac surgery (such as heart transplant) on patient reported symptoms. However, we feel that a survey of post-traumatic stress symptoms in a cohort of patients at risk for trauma and potential cognitive impairment, can provide researchers and clinicians knowledge regarding symptom burden and potential for intervention with this vulnerable population.

The goal of this retrospective study was to characterize the types and frequency of posttraumatic stress-related symptoms present in pediatric patients at the time of HT and assess needs in this cohort. Given the frequency of PTSS symptoms in our cohort (over one-third of the sample), there seems to be a high need for psychiatric care among pediatric heart transplant recipients. Currently, lack of mental health resources is a problem for pediatric and transplant patients and can impede on much needed psychiatric and/or psychological care. Useful interventions based on these data could include use of surveys and standardized quality of life forms given to pretransplant patients and their parents to elicit symptoms that occur commonly in these patients. Based on the responses, interventions can then be addressed and implemented pre-transplant with hopes that this will lead to better patient coping and, in turn, lead to better adherence post-transplant. The ultimate goal would be reduction in posttraumatic stress symptoms, through the identification and addressing of psychosocial issues, and continual quality improvement initiatives specifically geared towards pediatric heart transplant candidates and their families. 


\section{References}

1. Todaro JF, Fennell EB, Sears SF. Review: cognitive and psychological outcomes in pediatric heart transplantation. J Pediatr Psychol 2000;25:567-76.

2. Mintzer LL, Stuber ML, Seacord D, et al. Traumatic stress symptoms in adolescent organ transplant recipients. Pediatrics 2005;115:1640-4.

3. Stuber ML, Shemesh E. Posttraumatic stress disorder in medically ill patients: what is known, what needs to be deter- mined, and why is it important? CNS Spectr 2006;11;106-12.

4. Kazak AE, Alderfer MA, Streisand R, et al. Treatment of posttraumatic stress symptoms in adolescent survivors of childhood cancer and their families: a randomized clinical trial. J Fam Psychol 2004;18:493-504.

5. American Psychiatric Association. Diagnostic and statistical manual of mental disorders. 4th ed. Washington, DC: American Psychiatric Association; 2000.

6. Cavalcanti-Ribeiro P, Andrade-Nascimento M, Morais-de-Jesus M, et al. Post-traumatic stress disorder as a comorbidity: Impact on disease outcomes. Expert Rev Neurother 2012;12:1023-37.

7. Bunzel B, Laederach-Hoffman K. Solid organ transplantation: are there predictors for posttransplant noncompliance? Transplantation 2000;70:711-6.

8. Chartrand C, Servando ES, Chartrand S. Risk factors for acute rejection alter pediatric heart transplantation. Transplant Proc 2001;33:1732-4.

9. Steinberg AM, Brymer MJ, Kim S, et al. Psychometric properties of the UCLA PTSD reaction index: part I. J Trauma Stress 2013;26:1-9. 